\title{
'Optimum mobility' facelift. Part 2 - the technique
}

\author{
Nabil Fanous MD FRCSC ${ }^{1}$, Naznin Karsan MD ${ }^{1}$, Kristina Zakhary MD ${ }^{1}$, Carolyne Tawile MD ${ }^{2}$
}

\begin{abstract}
N Fanous, N Karsan, K Zakhary, C Tawile. 'Optimum mobility' facelift. Part 2 - the technique. Can J Plast Surg 2006;14(2):75-87.
\end{abstract}

\begin{abstract}
In the first of this two-part article on the 'optimum mobility' facelift, facial tissue mobility was analyzed, and three theories or mechanisms emerged: 'intrinsic mobility', 'surgically induced mobility' and 'optimum mobility points'.

In this second part, these three theories are applied to a rhytidectomy procedure termed 'optimum mobility' facelift. Before surgery, 'optimum mobility points' are marked on the skin. During surgery, the subcutaneous dissection is kept to a minimum by carrying it out precisely to these 'optimum mobility points'. The facial tissues, with their skin and superficial musculoaponeurotic system attachments intact, are then mobilized laterally using the 'intrinsic mobility' phenomenon, and this mobilization fixed in place using mattress sutures.

The 'optimum mobility' facelift is an efficient rhytidectomy technique that has a thoughtful, precise plan, a low complication rate, a fast recovery and very satisfactory results.
\end{abstract}

Key Words: Facelift; Optimum mobility; rhytidectomy

In the first part of the present study (pages 67-73), the theo$I_{\text {ries and mechanisms of facial tissue mobility were analyzed. }}$ In this second part, applying those principles to actual facelift surgery is discussed.

It seems reasonable to assume that if we can achieve such a remarkable facial tightening with just two fingers, then we should be able to do the same or better with limited surgical dissection.

In the present paper, a surgical technique termed 'optimum mobility' facelift is presented and the results are analyzed.

\section{'OPTIMUM MOBILITY' THEORIES APPLIED TO FACELIFT SURGERY}

To imitate the result obtained by a middle-aged woman using her fingers to show her surgeon what kind of a result she anticipates, one needs to analyze what actually happened beneath the woman's fingers and try to duplicate it surgically.

\section{Analyzing the fingers manoeuvre}

The patient's fingers manoeuvre, when reviewed in a slowmotion mode, may be divided into four stages:

- First, the woman chooses a point on her face;

- The woman then reaches with her fingers to that point (by placing her two fingers on it);

\section{Lifting du visage à " mobilité optimale " - Partie 2 : Technique}

Dans la première partie de cet article en deux volets sur la technique de lifting du visage à « mobilité optimale », l'auteur a présenté une analyse de la mobilité des tissus du visage et les trois théories ou mécanismes qui en découlent, soit la « mobilité intrinsèque ", la « mobilité induite chirurgicalement » et les " points de mobilité optimale ».

Dans la deuxième partie, ces trois théories sont appliquées à une intervention de rhytidectomie appelée lifting du visage à "mobilité optimale ». Avant la chirurgie, les « points de mobilité optimale » sont marqués sur la peau. Durant la chirurgie, la dissection sous-cutanée est réduite au minimum » car elle se limite précisément à ces « points de mobilité optimale ». Les tissus du visage, avec leurs points d'ancrage intacts avec les systèmes cutané et musculo-aponévrotique superficiel, sont ensuite mobilisés latéralement, en mettant en profit le phénomène de «mobilité intrinsèque » et une fois mobilisés, ils sont fixés en place à l'aide de sutures en matelas.

Le lifting du visage à " mobilité optimale » est une technique de rhytidectomie efficace qui repose sur un plan raisonné et précis, s'accompagne d'un taux de complications faible, permet une récupération rapide et procure des résultats très satisfaisants.

- The woman then mobilizes the facial tissues laterally and superiorly; and

- The woman fixes that obtained advancement by keeping her fingers in place.

\section{Duplicating the fingers manoeuvre surgically}

Now, to imitate the above four steps and implement them surgically, the following need to be performed:

\section{- Finding the 'optimum mobility points'}

Rather than choosing a point haphazardly on the face as the patient did, a more sophisticated 'optimum mobility point' is marked for each of the different parts of the face and neck.

As explained before, these 'optimum mobility points' should be ideally located to produce the maximal possible effect on the central face (through 'intrinsic mobility'), while being as close as possible to the incision (auricle, hairline). Advancing the pulling fingers farther medially beyond these points will have a negligible effect on the centre of the face and neck; hence, they are called the 'points of diminishing return'.

In actual surgery, three main 'optimum mobility points' (Figure 1, points A, B and C) are marked, one on each of the upper face, lower face and neck. These three 'optimum mobility points' are then connected to form an 'optimum mobility line' (Figure 1 - line BAC).

${ }^{1}$ Department of Otolaryngology, Head and Neck Surgery, McGill University; ${ }^{2}$ Department of Plastic Surgery, Charles LeMoyne Hospital, Montreal, Quebec

Correspondence: Dr Nabil Fanous, 1 Westmount Square, Suite 1380, Westmount, Montreal, Quebec H3Z 2P9. Telephone 514-935-9906, fax 514-935-6482, e-mail cosmeticsurgery123@videotron.ca 


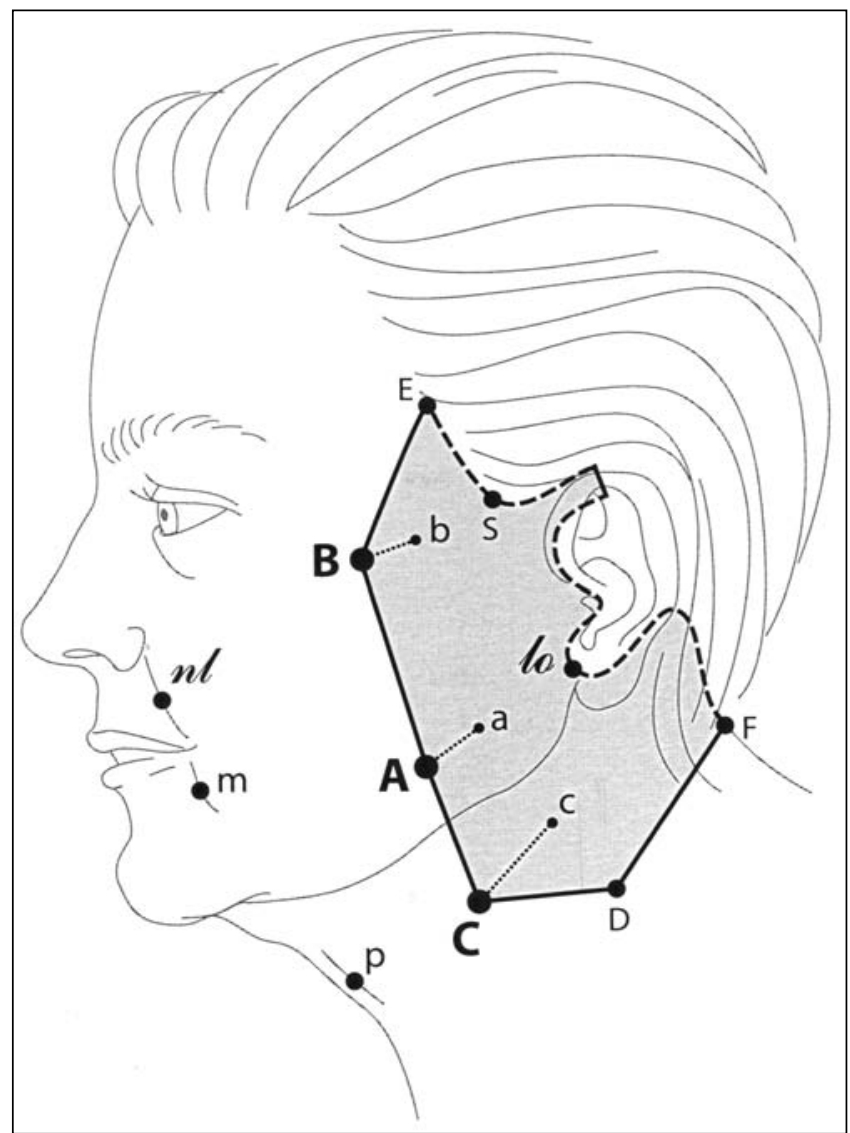

Figure 1) Preoperative marking of the 'optimum mobility' facelift. The three main 'optimum mobility points' (A for the lower face, B for the upper face and $C$ for the neck) are marked, and then joined together to form the 'optimum mobility line' BAC. This line is then connected to the upper end of the incision (E) with a straight line, and to the lower end of the incision (F) with $a V$-shaped line angled at D. Points $a, b$ and $c$ represent the first attempts of the surgeon's index finger to locate the three main 'optimum mobility points' A, B and C. The nasolabial fold is represented by $\mathrm{nl}$. The 'marionette' line is represented by $m$. The platysma bands are represented by $p$. The inferomedial angle of the sideburn is represented by s. The lobule is represented by lo. The incision is represented by the dashed line from $E$ to $F$

\section{- Reaching the 'optimum mobility points and line'}

Rather than reaching the 'optimum mobility points' with the fingers placed over the skin externally as the patient did, these points are reached internally by dissecting in the subcutaneous plane and elevating a skin flap. This dissection stops exactly at the 'optimum mobility line' (Figure 1 - line BAC).

\section{- Mobilization (laterally and superiorly)}

Rather than using the fingers externally for mobilization in a superolateral direction, a number of sutures are used internally to catch the superficial musculoaponeurotic system (SMAS) exactly at the 'optimum mobility points' and to pull it laterally and superiorly, with the skin still attached to it. A remarkable mobilization of the face and neck soft tissues is then witnessed, thanks to 'intrinsic mobility'.

\section{- Fixing the obtained mobilization}

In the patient's story, the result achieved by her 'fingers manoeuvre' will only last as long as her fingers are kept on the face (in other words, as long as the displacement is fixed in place). In surgery, this fixation is achieved by using slow absorbing or permanent sutures that pull and plicate the SMAS under high tension, for two months or longer, until it adheres to its new position.

This fixation of the gained mobilization is the last of the four important steps used to surgically duplicate the manoeuvre of the pulling fingers.

\section{SURGICAL TECHNIQUE OF THE 'OPTIMUM MOBILITY' FACELIFT}

To fully understand the 'optimum mobility' facelift technique, the above-mentioned four surgical steps will now be described in detail, as they are incorporated in an actual facelift procedure.

\section{Finding the 'optimum mobility points' (preoperative marking)}

Finding the 'optimum mobility points' is one of the two crucial parts of this technique. Three main 'optimum mobility points' (Figure 1, points A, B and C) are marked, one in three areas: the lower face, the upper face and the neck.

For the lower face, the index finger of the surgeon's right hand is placed just above the mandibular rim (Figure 1 - point a), approximately $1.3 \mathrm{~cm}$ to $2.5 \mathrm{~cm}$ (0.5 in to $1 \mathrm{in})$ in front of the lobule (Figure $1-l o$ ), anterior and inferior to it, while the surgeon's eyes are focused on the lower nasolabial fold and the mouth corner 'marionette' fold (Figure $1-n l$ and $\mathrm{m}$ ) to monitor the resultant improvement of the central lower face. The finger firmly pulls the skin laterally and superiorly (at approximately $30^{\circ}$ to $45^{\circ}$ ) until it meets increasing resistance and cannot move any further. As this is performed, a tightening of the lower nasolabial and 'marionette' folds is noticed. Then, this index finger is kept in place, while the index finger of the left hand lands on the skin just in front of it, and again pulls firmly in the same lateral superior direction. A further improvement is noticed on both folds medially. This technique of advancing index fingers is then repeated until the next resultant improvement detected on these two folds is negligible. A mark is then made at the site of the last placed finger (Figure 1 - point A) to identify the 'optimum mobility point' of the lower face, which is usually located between $2.5 \mathrm{~cm}$ and $5 \mathrm{~cm}$ (1 in to $2 \mathrm{in}$ ) in front of the lobule (or the future lower facelift incision).

In the upper face, the same technique is repeated to identify the second 'optimum mobility point'. The surgeon's right index finger is placed over the upper cheek and malar area (Figure 1 point b), approximately $1.3 \mathrm{~cm}$ to $2.5 \mathrm{~cm}(0.5$ in to $1 \mathrm{in})$ anterior and inferior to the angle of the sideburn (Figure 1 - point s), at a horizontal level, slightly higher than the superior limit of the tragus. The pull is again applied firmly in a superolateral direction and the upper nasolabial fold (Figure 1 - point $n l$ ) is monitored. Then, the other index finger is placed just anterior and a little inferior to the first one, and so on, etc, until the main 'optimum mobility point' of the upper face is found and marked (Figure 1 - point B), which is also usually located at $2.5 \mathrm{~cm}$ to $5 \mathrm{~cm}$ ( 1 in to 2 in) from the anterior inferior angle of the sideburn(s).

In the neck, the pulling index finger starts over the sternocleidomastoid muscle (Figure 1 - point c), approximately $2.5 \mathrm{~cm}$ to $3.8 \mathrm{~cm}$ ( 1 in to $1.5 \mathrm{in}$ ) below the mandibular edge, depending on how far down the neck laxity reaches. Then, the technique of advancing index fingers is repeated while 
monitoring the central neck folds and platysma cords (Figure 1 point $\mathrm{p}$ ), until the 'optimum mobility point' of the neck is found and marked (Figure 1 - point $\mathrm{C}$ ). This point usually falls on the inferior extension of the line BA (connecting the two 'mobility points' of the upper and lower face).

The three 'optimum mobility points' (Figure 1 - points B, A and $C$ ) are then connected to each other to form a line (BAC). This line, termed 'optimum mobility line', represents the ideal location of the mobilizing and fixing sutures. This 'optimum mobility line' also delineates the most anterior extent of the skin dissection that is necessary to give the surgeon access to place these fixing sutures.

The second phase in preoperative marking is to draw the incision line, which depends entirely on the surgeon's preference. The authors prefer a hairline incision along the sideburn, a post-tragal incision in the preauricular area, and a postauricular incision continuing along the neck hairline (Figure 1 dashed line $\mathrm{E}$ to $\mathrm{F}$ ). Other courses of incisions may alter the location of the postoperative scars or displace the hairline, but should not affect the efficiency of the 'optimum mobility' facelift.

The third and last phase of the marking is to connect the line BAC with the upper and lower ends of the rhytidectomy incision (Figure 1 - points $\mathrm{E}$ and $\mathrm{F}$ ). To join point $\mathrm{B}$ with the upper end of the incision (point $\mathrm{E}$ ), a straight line $\mathrm{BE}$ is drawn, which has usually an inclination of $30^{\circ}$ to $45^{\circ}$. As for point $\mathrm{C}$, it is joined with the most inferior end of the incision (point $\mathrm{F}$ ) via a V-shaped line CDF, angled at $\mathrm{D}$, with one arm $(\mathrm{CD})$ shorter than the other (DF). This configuration of skin dissection (CDF) gives the surgeon better access during surgery for placing the suture at point $\mathrm{C}$.

\section{Dissection}

The local anesthetic infiltration is performed as usual with a diluted lidocaine and epinephrine solution in the area of the skin flap (Figure 1 - perimeter EBACDF). Ideally, this infiltration should only extend $1 \mathrm{~cm}(0.4 \mathrm{in})$ or so medially anterior to this perimeter because too much infiltration beyond this line will balloon the medial facial tissues unnecessarily and limit their intrinsic mobility during surgery.

After the incision (Figure 1 - dashed line E to F) is made, a subcutaneous dissection is carried out precisely to the perimeter EBACDF. Hemostasis is achieved with electrocautery.

\section{Mobilization and fixation}

The combination of mobilization and fixation is the other crucial, important part of the technique. As shown in Figure 2, an average of eight mattress sutures are used along the 'optimum mobility line' and its extensions (EBACDF). These sutures may be semipermanent or permanent (the authors prefer polyglactin 910). Three key sutures are placed at the three main 'optimum points' (Figure 2 - points B, A and C), and five additional supporting sutures (Figure 2 - SS) are placed as follows: one at point $\mathrm{D}$, two between points $\mathrm{D}$ and $\mathrm{F}$, one between points $\mathrm{A}$ and $\mathrm{B}$, and one between points $\mathrm{B}$ and $\mathrm{E}$.

The first mattress suture is placed at point $A$. The surgeon catches the SMAS on the inside of that point, at the absolute end of the subcutaneous dissection. The first one-half of a horizontal mattress suture (Figure 2 - mattress suture at A, dot 1 to dot 2) is started by penetrating the SMAS at this point, in a superior to inferior direction (in the case of the left face), while trying to curve the needle forward and deep into the undissected

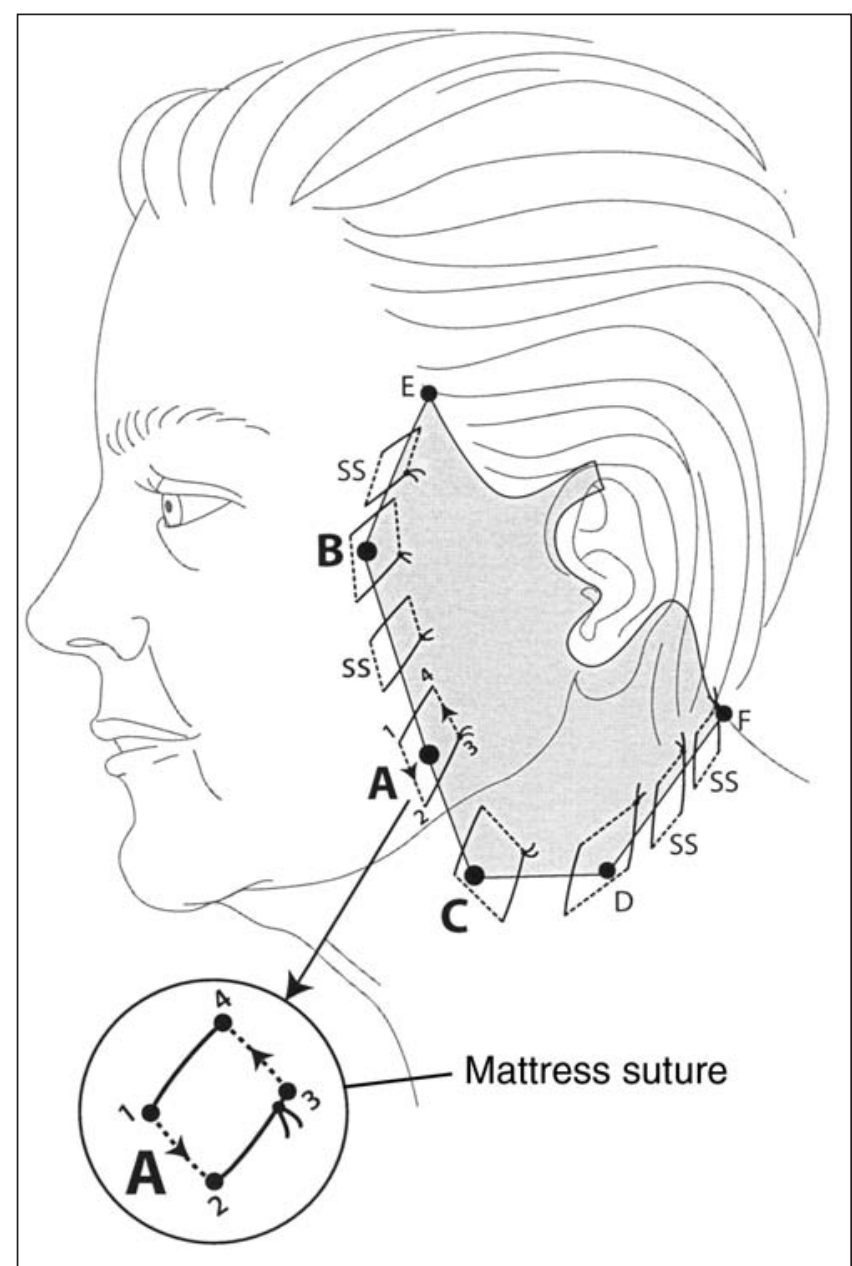

Figure 2) Mobilization and fixation of facial tissues with superficial musculoaponeurotic system (SMAS) plicating mattress sutures (left face). Three key sutures are placed in the SMAS at the three main 'optimum mobility points' A, B and C. Five additional supporting sutures ( $D$ and four SS) are then added, one at point $D$ and four along the perimeter EBACDF. These mattress sutures are all inclined superiorly and laterally $\left(30^{\circ}\right.$ to $\left.45^{\circ}\right), 1,2,3$ and 4 represent the numbered directions for the mattress sutures (please see text)

SMAS. The distance between dots 1 and 2 is maintained at approximately $13 \mathrm{~mm}$ (0.5 in). The second one-half of the suture (Figure 2 - mattress suture at point $\mathrm{A}$, dots 3 to 4 ) is then performed in an inferior to superior direction, taking a deep bite in the posterior SMAS at approximately $1.3 \mathrm{~cm}$ to $2.5 \mathrm{~cm}(0.5$ in to $1 \mathrm{in})$ lateral and superior $\left(30^{\circ}\right.$ to $\left.45^{\circ}\right)$ to the first one-half of the suture used. In the case of the right face, the opposite directions are used; inferior to superior for the first one-half of the suture, then superior to inferior for the second one-half of the suture.

The mattress suture $A$ is then tied under extreme force and secured with five knots. An impressive tightening is immediately observed at the lower nasolabial fold, the 'marionette' fold, the jowl and the mandibular line areas. A mild depression may be noted at the suture site, but is always temporary. A marked dimple, however, is a sign that the suture has been taken too superficially and that it should be repeated. 


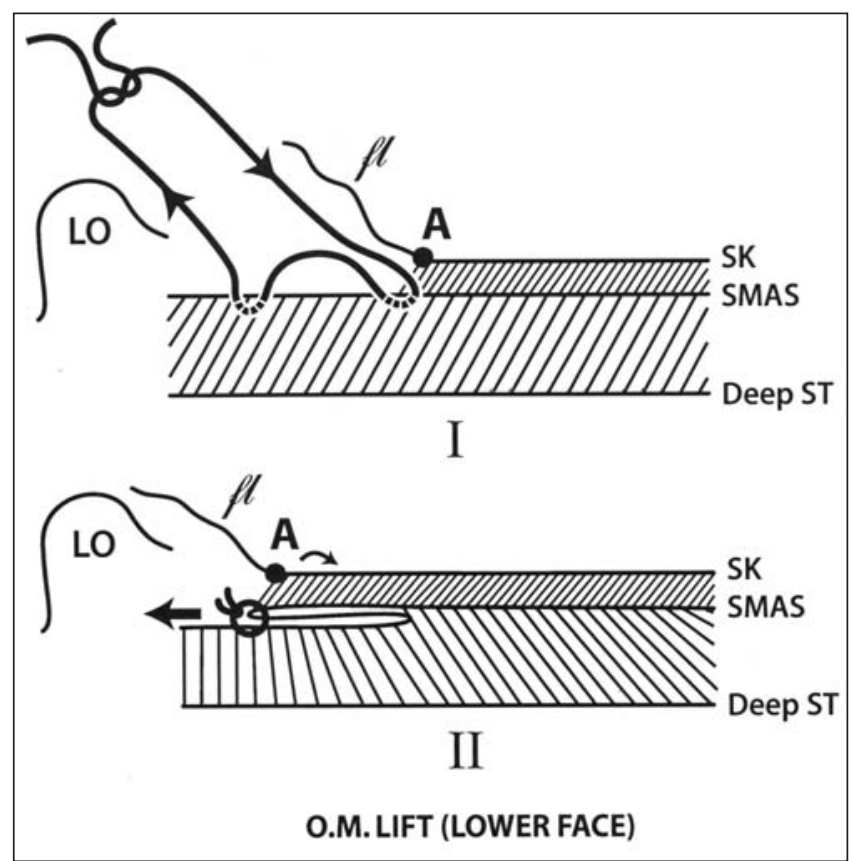

Figure 3) 'Optimum mobility' (OM) facelift technique in the lower face. I The superficial musculoaponeurotic system (SMAS) plicating mattress suture at the optimum mobility point A of the lower face is being placed. The first one-half of the mattress suture catches the SMAS underneath point A, at the base of the dissected flap (fl). II As the suture is tied, the SMAS and overlying skin are both mobilized laterally 'en bloc' (large arrow). However, a medial retrodisplacement of the skin takes place (small curved arrow), but is minimal because of the shortness of the septae in the sub-skin plane. Deep ST Structures (deep muscles, periosteum); LO Lobule; SK Skin

Once the mattress suture at point A is completed, the surgeon proceeds with the other mattress sutures below it in the neck (Figure $2-\mathrm{C}, \mathrm{D}, \mathrm{SS}$ and SS), and finally with the ones above it in the cheek and temple areas (Figure $2-\mathrm{SS}, \mathrm{B}$ and $\mathrm{SS})$. The total number of mattress sutures is usually around eight (Figure $2-B, A, C, D$ and four SS). In each one of these sutures, the second one-half of the mattress suture is taken at approximately $1.3 \mathrm{~cm}$ to $2.5 \mathrm{~cm}(0.5$ in to $1 \mathrm{in})$ in a lateral superior direction (at approximately $30^{\circ}$ to $45^{\circ}$ ) to the first one-half of the suture. Although all sutures are important, the vital ones are located at the three main points (Figure 2 points $\mathrm{B}, \mathrm{A}$ and $\mathrm{C})$. Furthermore, the surgeon may add one or two extra sutures along the EBACDF line whenever he or she feels that the tension after any suture is less than ideal.

\section{SUTURING TECHNIQUES OF THE UPPER FACE VERSUS THE LOWER FACE AND NECK}

A schematic representation of the 'optimum mobility' facelift technique in the lower face is shown in Figure 3.

As described above, in the case of the lower face, a skin flap (Figure 3I - fl) is raised to 'optimum point' A. Then, a mattress suture (Figure 3I - the loose suture not yet tightened) is used to plicate the SMAS at the limit of dissection (ie, on the inside

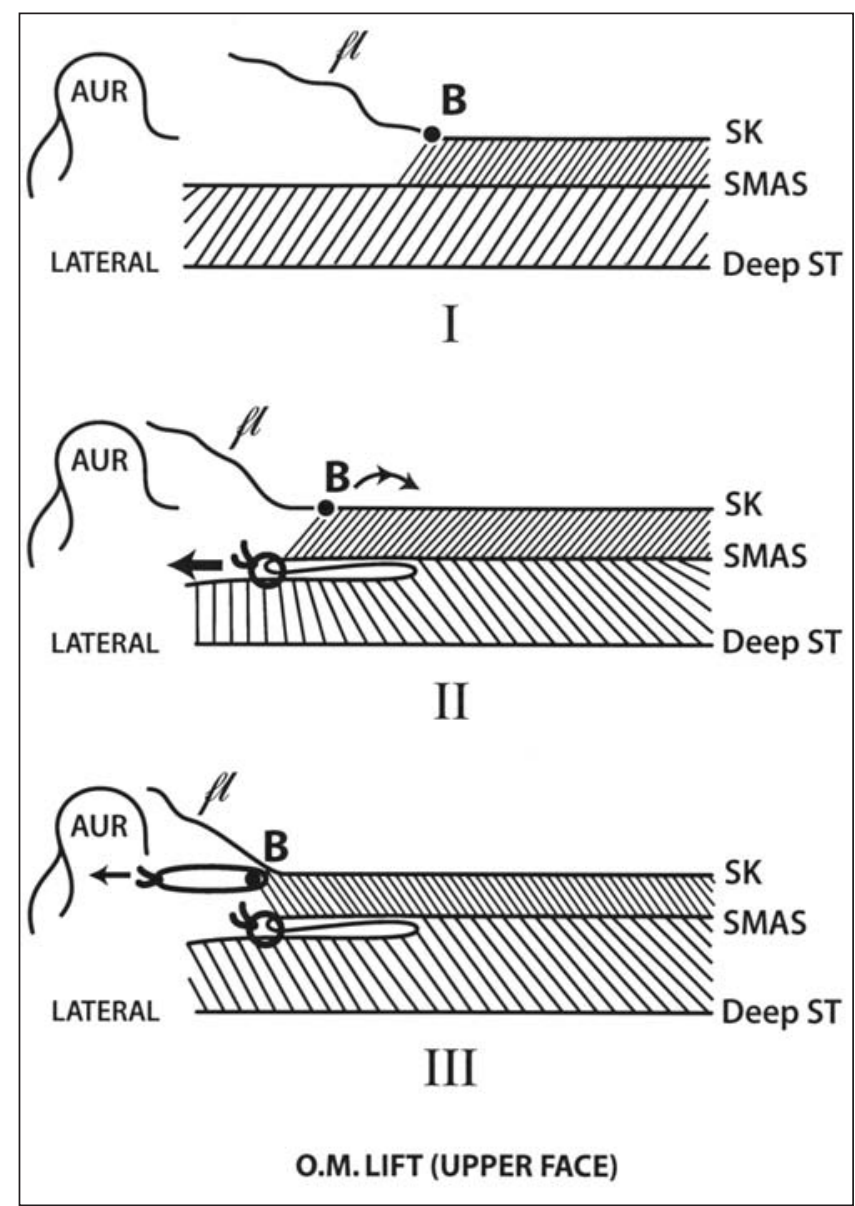

Figure 4) 'Optimum mobility' (OM) facelift technique in the upper face. I The subskin layer is thicker and fatter than that of the lower face; therefore, its septae are longer. II The superficial musculoaponeurotic system (SMAS) plicating suture at the optimum mobility point B of the upper face achieves a good lateral pull (straight bold arrow). However, the medial retrodisplacement of the skin (curved arrow) is more marked than that of the lower face because of the thicker subskin plane and its longer septae. III To improve the result and counter the skin retrodisplacement, an additional mattress suture (shown as an oval suture) is added at a more superficial level, into the subskin plane and just underneath the dermis at point B (arrow shows lateral pull). AUR Auricle; Deep ST Structures (deep muscles, periosteum); fl Flap; SK Skin

of that 'optimum point' A). Once the suture is tightened (Figure 3II - circular suture), the SMAS and the overlying skin still attached to it both move laterally 'en bloc' (Figure 3II large straight arrow). This marked mobilization of the SMAS and the skin attached to it is mostly due to the lateral rotation of septae in the sub-SMAS plane. By contrast, in the subskin plane, a backward medial rotation of the septae takes place (Figure 3II - small curved arrow). However, because these septae are short, the skin retrodisplacement is minimal and hardly affects the major advancement of the SMAS and skin by the mattress suture.

In the case of the upper face (Figure 4I), the subcutaneous plane is thicker and fatter, so the subskin septae are theoretically longer. The SMAS plicating suture at point B (Figure 2 and Figure 4II - circular suture) also achieves a good lateral pull 
(Figure 4II - large straight arrow). However, the retrodisplacement of the skin (backward rotation of the longer subskin septae, Figure 4II - thin curved arrow) is more marked than in the case of the lower face. So, to improve the result in this area of the upper face, an additional mattress suture is added at a more superficial level, into the subskin plane and just underneath the dermis at point B (Figure 4III - oval suture). On closing this second suture, further mobilization of the upper cheek skin should occur. The dimple caused by this additional suture is slightly more noticeable than the dimple of the deeper mattress suture, but is easily hidden temporarily by hair because of its proximity to the hairline.

In other words, two mattress sutures are used at point B of the upper face: a deep one into the SMAS (the usual one) and a superficial one into the subcutaneous/dermis junction (the additional one).

\section{FLAP TRIMMING AND CLOSURE}

The trimming of the redundant skin flap, as well as the type of closure used, depends on the surgeon's preference. For the incision closure, the authors use intracuticular sutures. Small dependent drains are left in the postauricular area for $24 \mathrm{~h}$, and a soft dressing (adhesive foam and gauze) is placed over the face and neck. As well, the authors routinely use intraoperative steroids and antibiotics, although their benefit may be debatable.

At the completion of the closure, the marked 'optimum points' on the skin are expected to have moved considerably toward the incision. In other words, the previously dissected skin flap along the incision line should now be much shorter. As a consequence, the dead space should become smaller. Occasionally, this dead space may be almost obliterated in the younger patient population with minimal to moderate laxity.

Figure 5 shows the preoperative marking and the successive surgical steps of the 'optimum mobility' facelift.

\section{POSTOPERATIVE CARE}

All patients are seen the day following surgery to withdraw the drains and to rule out hematomas. The gauze dressing is removed, but the adhesive foam is kept on for a week. Even in the immediate postoperative period, the edema is usually minimal in the exposed and undissected centre of the face and neck. However, mild to moderate irregularities caused by the mattress sutures may be present along the incision. Usually, these 'bumps' or 'dimples' are easily hidden by hair and gradually dissipate over two to four weeks. This fact should be explained to patients before surgery and is very well accepted by them, once they are reassured of the eventual 100\% disappearance of these dimples. The early tightness in the face, and especially in the neck, may seem excessive to the surgeon not used to this technique. This, however, is a welcomed sign and the skin will always relax somewhat within two to three days. Patients are usually delighted by the lack of swelling in the central face and neck, and are presentable 10 days to two weeks postoperatively with a proper hairstyle covering the incision lines.

\section{PATIENTS' DATA AND RESULTS}

The 'optimum mobility' facelift was performed on 182 patients by the same surgeon (NF). Patient age ranged from 45 to 71 years, with a mean age of 56 years. There were 165 women and 17 men. The follow-up ranged from six to 33 months, with an average of 16.3 months.
The most common complication was hematoma, which happened in five patients $(2.7 \%)$. Four of these cases were minimal, all occurring in the postauricular area, and were discovered on the first postoperative day. These small hematomas ( $2 \mathrm{cc}$ to $10 \mathrm{cc}$ ) were removed by withdrawal of the drain, then expression of the blood collection out through the $1 \mathrm{~cm}$ ( $0.4 \mathrm{in})$ drain opening in the posterior incision. A pressure dressing was then reapplied, and the four patients were seen every two days for one week. Only in two patients did the hematoma partially recollect beyond the week; the hematomas were left to liquefy and later suctioned with a needle. The fifth patient was healing well at the oneweek visit, but called later on the 13th postoperative day to complain about bleeding above the ear. When seen in hospital, she had blue-coloured swelling of the preauricular flap, as well as continuous bleeding from the sideburn incision. The patient was taken to the operating room and the bleeder (superficial temporal vein) was ligated. This was the only case in the author's patient series $(0.5 \%)$ who required going back to the operating room.

There were two cases of multiple suture rejections, one of silk sutures and the other of Ti•cron (Tyco, USA) (braided polyester fibre) sutures, and these occurred between two and 13 months postoperatively in the early cases of this series and prompted the change to the slowly absorbable sutures. Incidentally, the authors could not detect obvious clinical differences in the long-term results obtained by the permanent sutures versus those obtained by the slowly absorbable ones.

In one case, a patient came to the office two weeks postoperatively and was very distressed about a dimple in front of her right lobule (the point A suture). Reassurance proved futile. The dimple was easily relaxed by anesthetizing its spot, then using a number 18 needle and manipulating it underneath the skin to cut the suture. The release of the suture resulted in instant resolution of the dimple and a happy patient. At the time, the belief of the surgeon was that removing one suture out of eight would not have a noticeable impact on the final result. However, at her one-year postoperative visit, the patient's right lobule was noticed to be slightly pulled medially and inferiorly, a minor but nevertheless unaesthetic complication that was never noticed in any other patient in the author's series. This incidence demonstrated the crucial role of the mattress sutures during the early postoperative period.

There was one case of mild hypertrophic scars that were treated with intralesional steroids and silicone patches, and improved over time.

There were no cases of nerve injury, infection or seromas.

\section{ADVANTAGES AND DISADVANTAGES OF THE 'OPTIMUM MOBILITY' FACELIFT}

The 'optimum mobility' facelift has many attractive features. It is a minimally invasive procedure. The theories of 'intrinsic mobility' and 'optimum mobility points' allow for the maximum possible result with the least possible dissection, which increases the efficiency and safety of the procedure, while reducing the operating time, as well as the intraoperative and postoperative edema and bleeding. The dead space, which is already small to moderate at the beginning of the procedure, is even smaller at the end. The resulting short skin flap means better vascularization and less likelihood of skin necrosis, an 

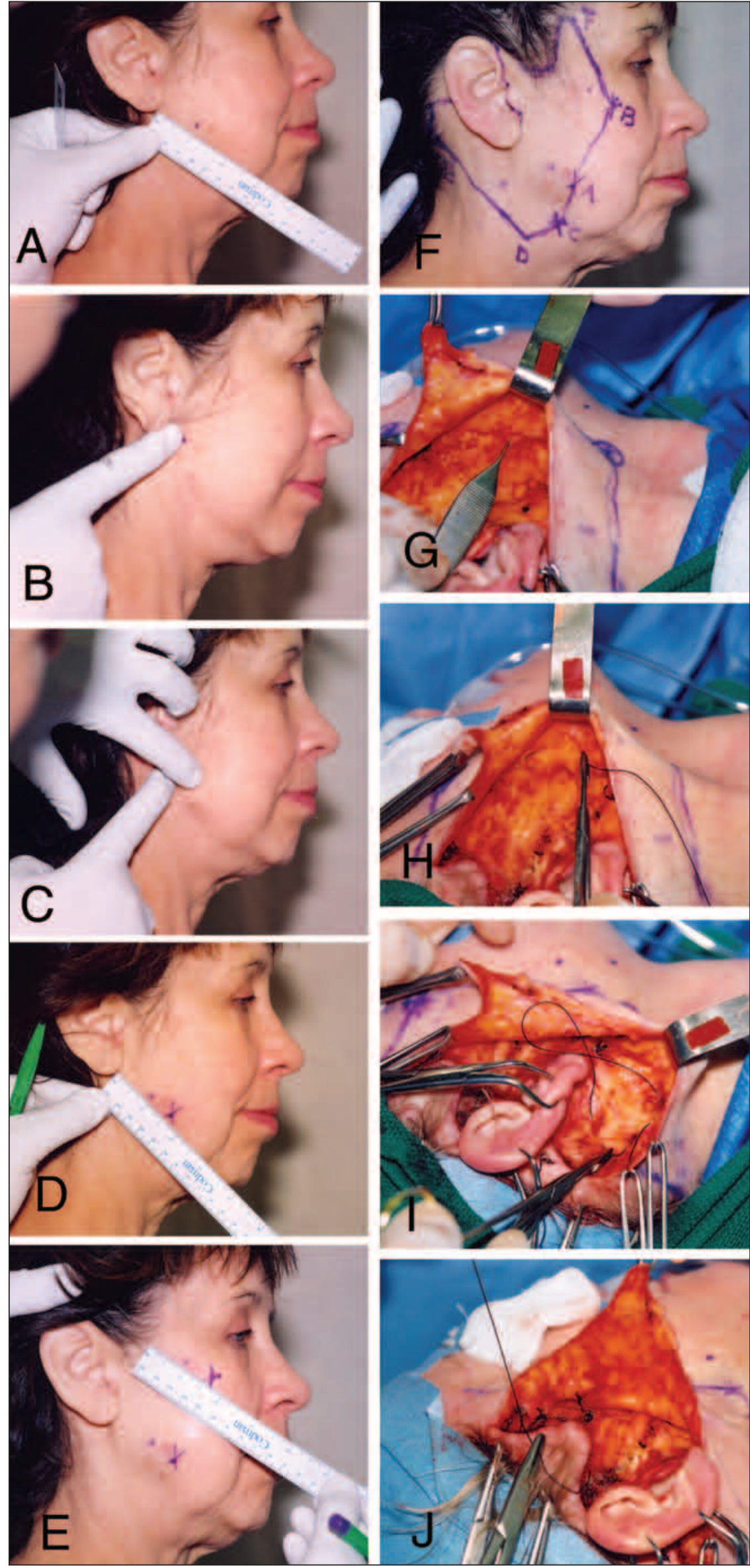

Figure 5) Successive preoperative and intraoperative steps of the 'optimum mobility' facelift. A Preoperative marking of the 'optimum mobility point' A of the lower face. A dot is marked on the skin just above the mandibular rim, approximately $1.3 \mathrm{~cm}$ to $2.5 \mathrm{~cm}(0.5$ in to $1 \mathrm{in})$ in front of the lobule. B The index finger of the surgeon's right hand is placed on the dot, while the surgeon's eyes are focused on the lower nasolabial fold and the 'marionette' fold, to monitor the resulting improvement on the central lower face. The finger firmly pulls the skin laterally and superiorly until it meets increasing resistance and cannot move any further. C This index finger is kept in place, while the index finger of the left hand lands on the skin just in front of it, and again pulls firmly in the same lateral superior direction. This technique of advancing index fingers is repeated until the next resultant improvement on the folds of the lower central face is negligible. D A mark $(x)$ is then made at the site of the last placed finger to identify the 'optimum mobility point' A of the lower face, which is usually located between $2.5 \mathrm{~cm}$ and $5 \mathrm{~cm}$ ( 1 in and 2 in) anterior and inferior to the lobule. $\mathrm{E}$ In the upper face, the same technique is repeated to identify the second 'optimum mobility point' B, usually located as well between $2.5 \mathrm{~cm}$ and $5 \mathrm{~cm}$ ( 1 in and 2 in) anterior and inferior to the angle of the sideburn. F In the neck, the third main 'optimum mobility point' $\mathrm{C}$ is identified using the same advancing fingers technique. This point usually falls on the inferior extension of the line BA. The line BAC, connecting the three main 'optimum mobility points', is termed the 'optimum mobility line'. It delineates the most anterior extent of the skin dissection needed to give the surgeon enough access to place the future mobilizing and fixing mattress sutures into the superficial musculoaponeurotic system (SMAS) along that line. The 'optimum mobility line' BAC is connected to the lower end of the incision (E in Figure 1) via a $V$-shaped line angled at $D$, and to the upper end of the incision (F in Figure 1) with a straight line. G Before placing the first mattress suture A of the lower face, the surgeon catches the SMAS on the inside of that point, located at the absolute end of the dissection. Notice the line of demarcation, a kind of a groove, along the limit of the dissection, between the firm-looking undersurface of the skin flap and the softer-looking surface of the SMAS. $\mathrm{H}$ The first one-half of a mattress suture in the right face, being placed deeply into the SMAS, exactly at the demarcation groove at the end of the flap dissection, at the 'optimum mobility line'. I The second one-half of a mattress suture in the right neck is being placed, lateral and superior to the first one-half of the suture, with an inclination of $30^{\circ}$ to $45^{\circ}$. J The uppermost mattress suture in the upper face is being completed. Notice the resultant narrowing of the dead space 
important issue when dealing with certain patients, such as diabetics or smokers. Because of the restricted final dead space, any potential bleeding is contained and cannot expand, which is not the case when large skin flaps are dissected over the neck or face. The chance of facial nerve injury is extremely remote. Most importantly, the 'optimum mobility' facelift gives surprisingly durable results, thanks to the very negligable postoperative relapse of the medial fascial tissues.

There are disadvantages, however, to this procedure. A good part of the result depends on the proper marking of the 'optimum mobility points' and the proper placement of sutures. The first few procedures may therefore be time-consuming. As well, the suture irregularities in front of the incision line, even though they are always temporary, need to be camouflaged by hair in the early postoperative period.

\section{DISCUSSION}

Optimum mobility versus other facelift techniques

The comparison between the 'optimum mobility' facelift and other popular rhytidectomy techniques (subcutaneous, subcutaneous with or without SMAS manipulation, sub-SMAS) may help clarify the advantages and disadvantages of this approach, as well as of the other approaches.

The subcutaneous lift

The surgical procedure of the subcutaneous lift is simple and the intraoperative improvement is satisfactory, but the result is short-lived. It is important to understand the dynamics of the subcutenous lift. A skin flap of variable length is raised (Figure 6A - fl). During the trimming of the excess skin, as one pulls on the lateral end of the skin flap (Figures $6 \mathrm{~A}$ and $6 \mathrm{~B}-$ point $\mathrm{L}$ ), the flap first advances by stretching between its most lateral point $\mathrm{L}$ and its most medial point $\mathrm{M}$, due to the 'surgically acquired mobility' secondary to flap dissection. Once it is fully stretched, the flap starts pulling the undissected skin and the SMAS, through 'intrinsic mobility', at its medial end (Figure 6B-point $\mathrm{M}$ ).

The first problem with this subcutenous lift is its variable area of dissection. The extent of skin dissection differs from one surgeon to another, depending on personal convictions regarding the benefits of a short flap (short flap dissection is safe, fast, etc) or of a long one (extensive dissection reaches the problematic medial laxity, the more the dissection the better the mobilization, etc).

The second and more serious problem with the subcutaneous lift is its poor dynamics. The main pulling force is applied to the most lateral end of the skin flap (Figure $6 \mathrm{~B}$ - point $\mathrm{L}$ ) at the closure line. This pulling force is transmitted to a faraway point at the medial end of the flap (Figure $6 \mathrm{~B}$ - point M), the one that truly carries the load. As discussed before, dissection of any flap leads to partial weakening and further stretching of that flap. Because the skin flap between points $\mathrm{L}$ and $\mathrm{M}$ has been dissected, it is now liberated from the restricting effect of the underlying septae in the subskin layer, and can therefore expand as the surgeon pulls it and then resects it before closure (Figure 6B). Furthermore, postoperatively, this expansion does not stop. The skin flap will continue to further stretch under the effect of gravity on its medial point $\mathrm{M}$ (Figure $6 \mathrm{C}$ - wavy arrow), leading to a slow medial retrodisplacement of point $\mathrm{M}$, and with it the partial relapse of the central facial tissues. In other words, the wider the original skin dissection (distance LM), the weaker and the more stretchable the flap will be

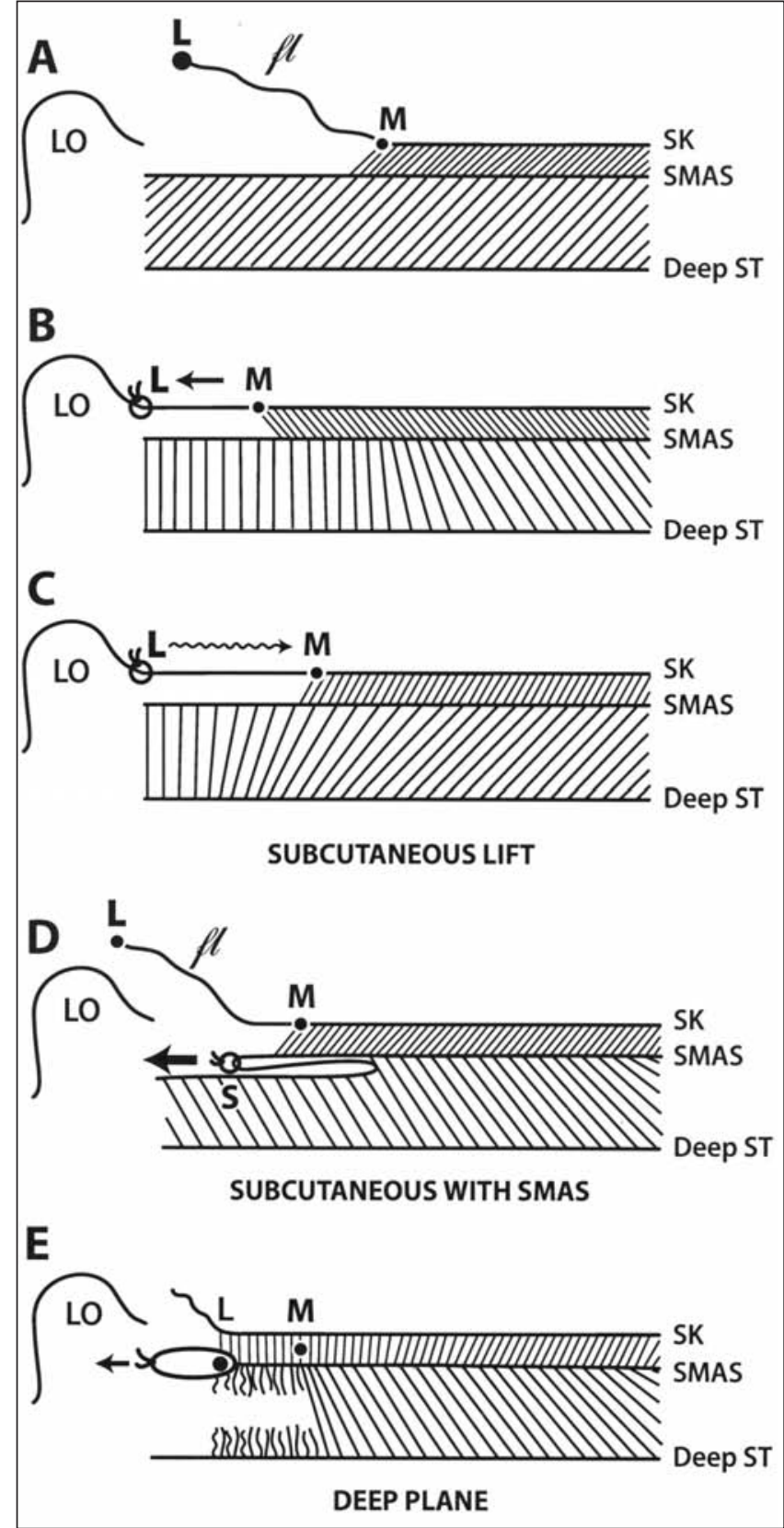

Figure 6) The subcutaneous lift. A A skin flap (fl) is raised. B The fl is pulled (arrow), trimmed and closed (circular suture). C The fl (between its medial end $\mathrm{M}$ and its lateral end $L$ ) is weakened and is free to stretch because of the absence of the underlying septae. Postoperatively, it continues to further stretch (wavy arrow) under the effect of gravity at its medial point $\mathrm{M}$, leading to the slow medial retrodisplacement of point $\mathrm{M}$ and to the partial relapse of the central facial tissues. D The subcutaneous lift with superficial musculoaponeurotic system (SMAS) manipulation. The $\mathrm{fl}$ is dissected. SMAS plication is then performed somewhere within the dissected area (circular suture). This SMAS tightening (arrow) creates a more efficient point of pull (point S) than that of the subcutaneous lift $(\mathbf{C}$ - point $\mathbf{M})$. This is because the pulled SMAS at point $S$ is only dissected superficially and is much stronger when compared with a skin flap. However, the exposed SMAS between the pulling suture and the end of the dissection (distance SM) is still susceptible to postoperative distension. E The sub-SMAS (deep plane) lift. A composite flap ( $L$ to $M$ ) of skin, subskin and SMAS layers is created via sub-SMAS dissection. Because of its thickness and the unviolated attachments between its skin and SMAS layers, this flap can better withstand the forces of tension and gravity. Deep ST Structures (deep muscle, periosteum); LO Lobule; SK Skin 
postoperatively, leading to the progressive descent of the central facial tissues.

The late postoperative result, in six months or more, is therefore expected to deteriorate, because the effective pull at point $\mathrm{M}$ is slowly diluted by the relaxation of the skin flap lateral to it, due to the unrelenting gravity force medial to it.

\section{The subcutaneous lift with SMAS manipulation}

SMAS plication (the SMAS is advanced and folded without being incised) and SMAS imbrication (the SMAS is advanced and superimposed after being incised and underlined) are two variations of SMAS displacement. When combined with a skin flap in a rhytidectomy, either technique takes the tension off the skin flap. Figure 6D shows the two parts of this procedure, mainly skin flap dissection (Figure $6-$ $f l)$ followed by SMAS manipulation (plication) somewhere within the dissected area (Figure 6D - circular suture). This SMAS tightening creates a new point of pull (point $S$ ) that is much stronger and more effective than the one of the subcutaneous facelift (Figure $6 \mathrm{~B}$ - point $\mathrm{M}$ ) for two reasons: 'intrinsic mobility' created with SMAS advancement is, as explained before, much more significant than the one created with skin advancement; and the pulled SMAS at point $\mathrm{S}$ (Figure 6D) is much less likely than the skin flap (Figure 6C) to distend postoperatively because the SMAS is stronger than a skin flap, and because it is only dissected superficially (Figure 6D - distance S to M), while the septae connecting its deep surface to the deep structures are still intact. This is why the long-term result of this procedure is superior to that of the subcutaneous lift alone.

However, the subcutaneous lift with SMAS manipulation has its drawbacks. The extent of skin dissection is again based on personal preferences rather than on the conscious analysis of tissue mobility. More important, there is a distance between the SMAS suture (Figure 6D - point S) and the end of the skin dissection (Figure 6D - point $\mathrm{M}$ ). The SMAS between these two points ( $S$ to $M$ ), because of being detached superficially from the skin, is susceptible to some postoperative distension. Theoretically, the longer the distance between points $\mathrm{S}$ and $\mathrm{M}$, the more relapsed the medial facial tissues will be.

\section{The sub-SMAS lift (deep plane lift)}

The main contribution of the deep plane technique is the creation of a composite flap (skin, subskin and SMAS) through sub-SMAS dissection (Figure 6E). Because of its thickness, and because of the unviolated attachments between its skin and SMAS layers, this flap is a resilient structure. It withstands high tension, while suffering minimal stretching between the most lateral pulling point (Figure $6 \mathrm{E}$ - point $\mathrm{L}$ ) and its most medial pulling point (Figure $6 \mathrm{E}$ - point $\mathrm{M}$ ). This is believed to give the deep plane lift technique the advantage of a long-term effect, with a limited stretching back of tissues postoperatively.

However, sub-SMAS dissection has some disadvantages, including some postoperative flap relapse, the possibility of facial nerve injury or synkinesis, and a demanding learning curve.

\section{THE ‘OPTIMUM MOBILITY’ LIFT}

The 'optimum mobility' facelift is not just another version of a short flap lift with SMAS tightening. Although, at first glance, both the 'optimum mobility' lift (Figure 3II) and the subcutaneous lift with SMAS manipulation (Figure 6D) seem similar, they differ in the two crucial ways listed, which are also the two important contributions of the 'optimum mobility' approach to the surgery of facial rejuvenation:

\section{- The idea of finding the 'optimum mobility points' (taking advantage of the 'intrinsic mobility' phenomenon)}

Finding the 'optimum mobility points' is the first unique characteristic of the 'optimum mobility' technique. These 'optimum mobility points' are not chosen haphazardly, but are meticulously planned to achieve the maximum possible mobilization of the undissected tissues medial to them, but with the minimum possible subcutaneous dissection lateral to them. They indicate the exact extent of the skin flap dissection. The efficiency of the 'optimum mobility' facelift truly depends on the accurate marking of the 'optimum mobility' points.

\section{- The idea of placing the pulling sutures at the absolute end of the dissection (at the 'optimum mobility points' and 'optimum mobility line')}

Placing the pulling sutures at the absolute end of the dissection is the second unique feature of the 'optimum mobility' technique. The SMAS tightening sutures are placed at the absolute end of the subcutaneous dissection, in other words at the 'optimum mobility points' (Figure 3), where the layers (skin and SMAS) and their sublayers (subskin and sub-SMAS) are still intact and attached to one another. Placing the SMAS pulling sutures at the end of the skin dissection, without any separating distance, leads to the 'en bloc' pulling of the medial facial soft tissues as one powerful mass, using the maximum possible 'intrinsic mobility', while restricting the stretching back phenomenon to the very minimum in the future. The value of this idea may be easily tested by any surgeon during any rhytidectomy procedure; the surgeon first places the plicating SMAS suture $2.5 \mathrm{~cm}$ to $5 \mathrm{~cm}$ ( 1 in to 2 in) behind the end of the skin dissection, then watches the tightening effect on the medial face. The suture is then removed and repeated at the absolute end of the dissection, at the 'optimum mobility line'. The difference is always remarkable.

Figures 7 to 11 show pre- and postoperative results of the 'optimum mobility' facelift.

\section{CONCLUSIONS}

The 'optimum mobility' facelift is a technique developed on the basis of three theories: 'intrinsic mobility', 'surgically-induced mobility' and 'optimum mobility points'. The surgical technique is characterized by two main ideas: locating the 'optimum mobility points' and placing the pulling sutures at the absolute end of the dissection.

The 'optimum mobility' facelift is started by locating these 'optimum mobility points', which are then used to delineate the limits of the subcutaneous dissection. The lateral mobilization of the facial tissues, via their 'intrinsic mobility', is then achieved and fixed with mattress sutures, placed at the absolute end of the dissection, along the 'optimum mobility line'.

The 'optimum mobility' facelift is a safe and efficient technique with low morbidity and a high satisfaction rate.

ACKNOWLEDGEMENTS: The authors thank Ildico Horvath, medical artist, Montreal General Hospital (Montreal, Quebec), for her assistance in preparing the artwork; Barbara Armbruster MA for editing; Amanda Fanous for the literature search; Stephanie Luetticken MBA for organizing the manuscript; and Joelle Bejjani for typing. 

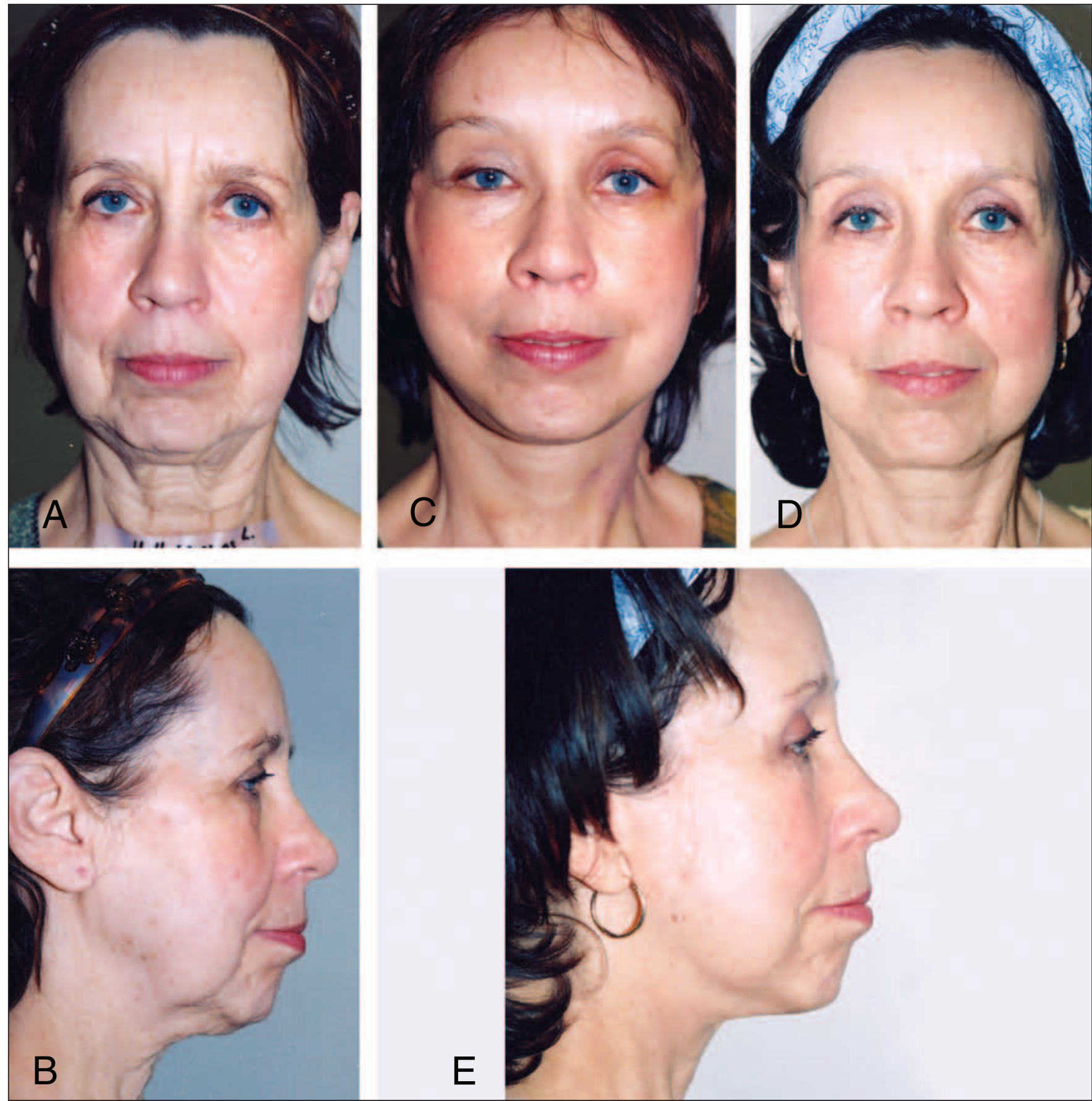

Figure 7) A and B are preoperative views of a 67-year-old patient with marked neck laxity and folds, as well as brow ptosis. This is the same patient depicted preoperatively in Figure 5. C Postoperative view six days after 'optimum mobility' rhytidectomy and a 'modified minimal-incision' forehead lift. $\mathbf{D}$ and $\mathbf{E}$ are postoperative results 12 months following surgery. Notice the persistent neck improvement one year later. No surgery was performed on the medial platysmal bands. Note: Since performing the 'optimal mobility' facelift, the author has not used the direct approach to the platysmal band through the submental incision, not even once 

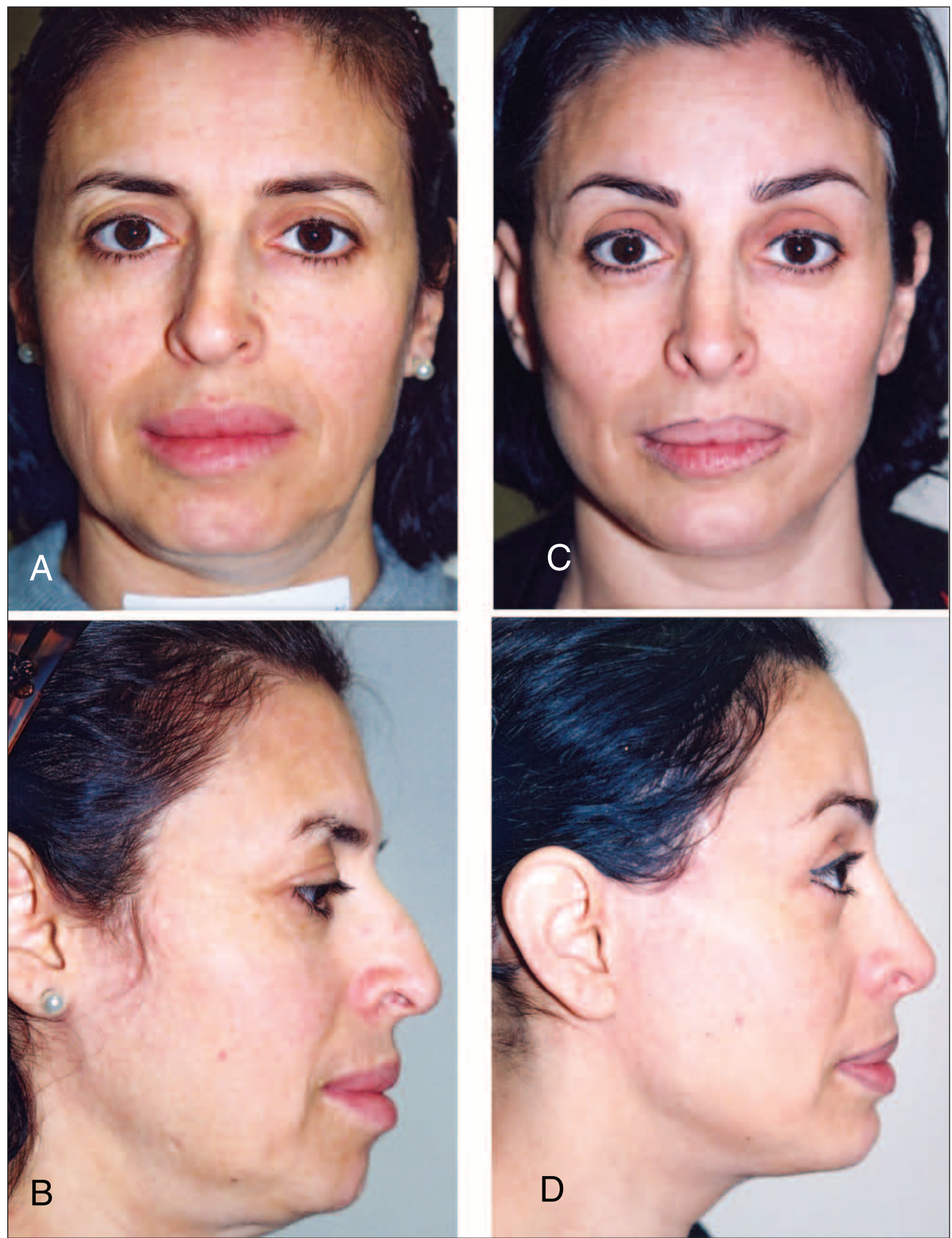

Figure 8) A and B are preoperative views of a 45-year-old patient requesting the correction of her droopy brows and her facial and cervical folds, as well as improving the appearance of her nose. $\mathbf{C}$ and $\mathbf{D}$ are postoperative views 14 months following 'optimum mobility' rhytidectomy, 'modified minimal-incision' forehead lift, septorhinoplasty and chin augmentation 

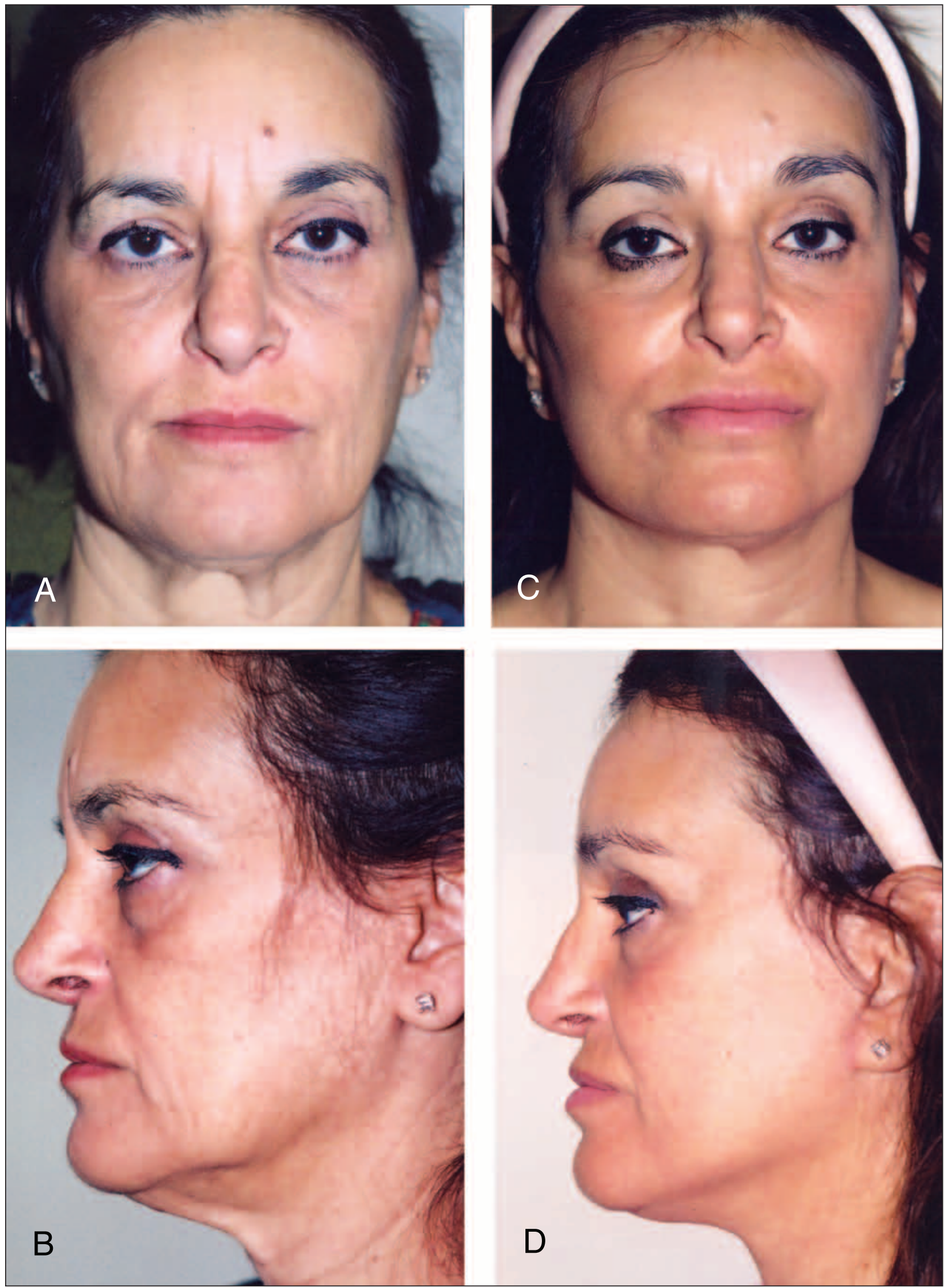

Figure 9) A and B are preoperative views of a 57-year-old patient with a general laxity of the face and neck, as well as forehead ptosis. C and D are postoperative views six months following 'optimum mobility' rhytidectomy and a 'modified minimal-incision' forehead lift. There was no surgical intervention in the submental area 


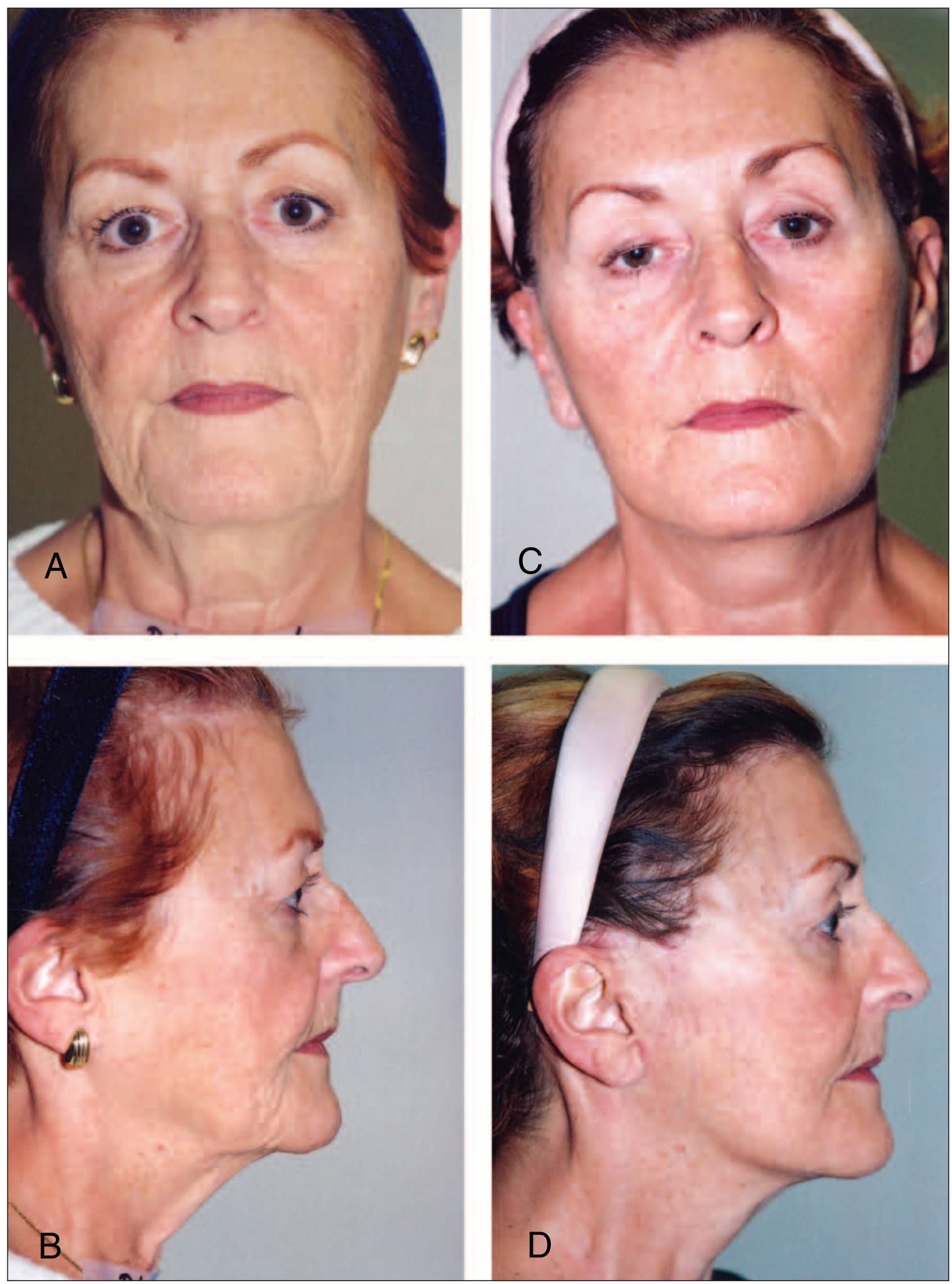

Figure 10) $\mathbf{A}$ and $\mathbf{B}$ are preoperative views of a 62-year-old patient with cervical platysmal bands and jowls. $\mathbf{C}$ and $\mathbf{D}$ are postoperative results eight months following 'optimum mobility' rhytidectomy and a 'modified minimal-incision' forehead lift. No platysmal work was performed in the central neck 

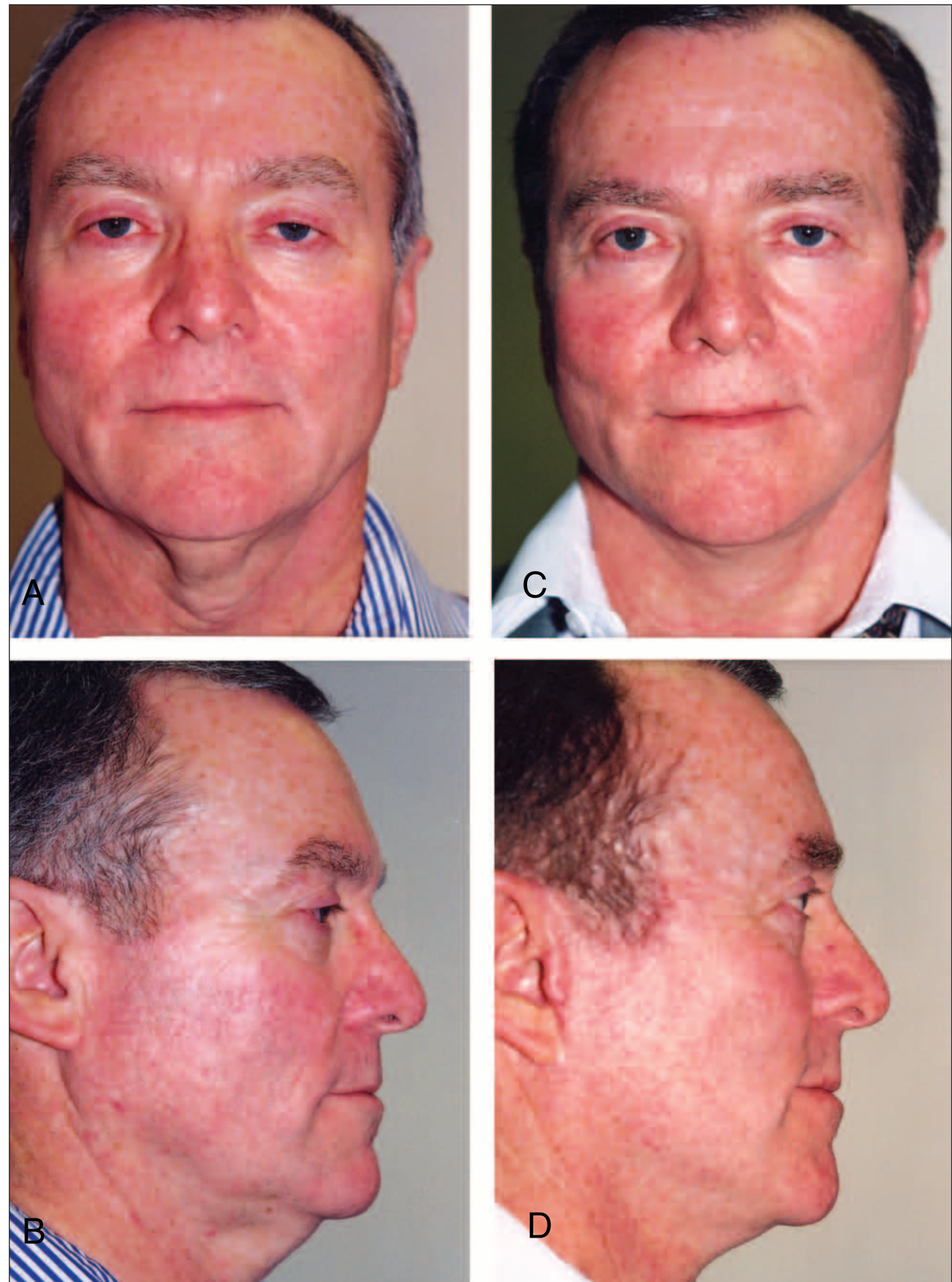

Figure 11) A and B are preoperative views of a 58-year-old patient who complained of a marked central neck laxity (turkey-neck deformity) and facial aging. $\mathbf{C}$ and $\mathbf{D}$ are postoperative views 14 months following 'optimum mobility' rhytidectomy (the forehead was not operated on). The improvement and durability of the central neck appearance were achieved without midline skin excision or platysmal manipulation 